\title{
Acetylcholine Attenuates Hypoxia/ Reoxygenation-Induced Mitochondrial and Cytosolic ROS Formation in H9c2 Cells via M2 Acetylcholine Receptor
}

\author{
Yi Miao $^{\mathrm{a}}$ Jun Zhou ${ }^{\mathrm{a}}$ Mei Zhao Jinjun Liu Lei Sun Xiaojiang Yu Xi He \\ Xiaoyue Pan Weijin Zang
}

Department of Pharmacology, Xi'an Jiaotong University, College of Medicine, Xi'an, aBoth authors contributed equally to this work

\section{Key Words}

Acetylcholine - Hypoxia/reoxygenation - Reactive oxygen species - Electronic transport chain - NADPH oxidase $\cdot$ Xanthine oxidase $\cdot M 2$ acetylcholine receptor

\begin{abstract}
Background: The anti-inflammatory and cardioprotective effect of acetylcholine (ACh) has been reported; nevertheless, whether and how ACh exhibits an antioxidant property against ischemia/reperfusion (I/R)-induced oxidative stress remains obscure. Methods: In the present study, H9c2 rat cardiomyocytes were exposed to hypoxia/reoxygenation $(H / R)$ to mimic $I / R$ injury. We estimated intracellular different sources of reactive oxygen species (ROS) by measuring mitochondrial ROS (mtROS), mitochondrial DNA (mtDNA) copy number, xanthine oxidase (XO) and NADPH oxidase (NOX) activity and expression of rac 1 . Cell injury was determined by lactate dehydrogenase (LDH) release and cleaved caspase- 3 expression. The siRNA transfection was performed to knockdown of M2 acetylcholine receptor (M2 AChR) expression. Results: 12-h hypoxia followed by 2-h reoxygenation resulted in an abrupt burst of ROS in H9c2 cells. Administration of ACh reduced the levels of ROS in a concentrationdependent manner. Compared to the H/R group, ACh decreased mtROS, recovered mtDNA copy number, diminished XO and NOX activity, rac 1 expression as well as cell injury. Cotreatment with atropine rather than hexamethonium abolished the antioxidant and cardioprotective effect of ACh. Moreover, knockdown of M2 AChR by siRNA showed the similar trends as atropine co-treatment group. Conclusions: $\mathrm{ACh}$ inhibits mitochondria-, XO- and NOX-derived ROS production thus protecting H9c2 cells against H/R-induced oxidative stress, and these beneficial effects are mainly mediated by M2 AChR. Our findings suggested that increasing ACh release could be a potential therapeutic strategy for treatment and prevention of $\mathrm{I} / \mathrm{R}$ injury.

Copyright (C) 2013 S. Karger AG, Basel
\end{abstract}


Miao/Zhou/Zhao et al.: ACh Reduces ROS Production via M2 AChR

\section{Introduction}

Ischemia/reperfusion (I/R) is an important clinical problem associated with procedures including angioplasty, thrombolytic therapy, and coronary bypass surgery, which is considered to induce additional lethal injury beyond a certain critical period [1]. A vast majority of studies support the view that oxidative stress due to a burst of reactive oxygen species (ROS) during reperfusion is the critical trigger of I/R injury [2]. In general, modest increases in ROS production and activation of ROS-dependent pathways exert cardioprotective effects [3]; however, excess ROS formation is detrimental. This is not only because they cause nonspecific oxidative damage to DNA, proteins and lipids, but also by modulating redox-regulated signaling cascades, which cause further damage and ultimately cell death[4].

In view of the detrimental role of ROS overproduction, it is logically to suggest that the application of antioxidants should be useful. Nevertheless, the clinical trial results with various antioxidants are disappointing [5]. Therefore, inhibiting the formation of ROS instead of scavenging these harmful species after their emergence may be more promising. The resources of ROS are multiple, of note, the mitochondrial electronic transport chain (ETC), NADPH oxidase (NOX) and xanthine oxidase (XO) are reported to be the main resources of ROS in the cardiomyocytes during pathophysiology process [6]. Moreover, numerous studies have shown that inhibiting these pathways by pharmacological strategies exhibits beneficial effect against cardiovascular diseases [7]. Unfortunately, to date many of these inhibitors have not been employed in patients due to their toxcity.

Recently, cumulative evidence suggests that vagus nerve stimulation (VNS) decreases infarct size, inflammatory markers and prevents myocardial remodeling in the modle of I/R $[8,9]$, but the potential mechanisms remain to be elucidated. Interestingly, Takaki Tsutsumi et al. demonstrated that VNS suppresses the level of myocardial ROS via muscarinic receptor (M receptor) in a murine model of chronic heart failure [10]. Besides, acetylcholine (ACh), the principle neurotransmitter of vagus nerve, reduces $\beta$-adrenoceptor and hypoxia-induced ROS $[10,11]$. Recent studies in our laboratory have shown that ACh reduces angiotensin II and hypoxia-induced ROS in H9c2 cells via M AChR $[12,13]$. These data suggest that ACh has the antioxidant property and this particular effect is likely to be also beneficial to attenuate myocardial I/R injury. Previous studies provide little information on whether and how ACh reduces the level of ROS during I/R in cultured cardiomyocytes. This study aims to address this by investigating the antioxidant effect of ACh during hypoxia/reoxygenation (H/R) and the potential mechanism.

\section{Materials and Methods}

\section{Cell lines, cell culture, cell treatment}

H9c2 cells, derived from rat embryonic ventricular cardiomyocytes, were purchased from American Type Culture Collection (ATCC). Cells were routinely grown in DMEM (Hyclone) supplemented with $10 \%$ fetal bovine serum (FBS; Hyclone), $100 \mathrm{U} / \mathrm{ml}$ penicillin (Sigma) and $100 \mu \mathrm{g} / \mathrm{ml}$ streptomycin (Sigma) at $37^{\circ} \mathrm{C}$ in a humidified atmosphere of $5 \% \mathrm{CO}_{2}$, and were fed every $2-3 \mathrm{~d}$ and were split 1 to 2 at $80 \%$ confluence. For experiments, cells were cultured for $24 \mathrm{~h}$ and were serum starved for $8 \mathrm{~h}$, then subjected to modified ischemia-mimetic solution and hypoxia for $12 \mathrm{~h}$ in a modular incubator gassed with $2 \% \mathrm{O}_{2}, 5 \% \mathrm{CO}_{2}$, and $93 \%$ $\mathrm{N}_{2}$ as previously described [13]. For reoxygenation, cells were removed and incubated in serum-free DMEM at $37^{\circ} \mathrm{C}$ in a humidified atmosphere of $5 \% \mathrm{CO}_{2}$ for $1 \mathrm{~h}, 2 \mathrm{~h}, 4 \mathrm{~h}, 8 \mathrm{~h}, 16 \mathrm{~h}$ and $24 \mathrm{~h}$ respectively.

To investigate the effect of ACh on ROS, cells were treated with various concentrations of ACh $\left(10^{-7}\right.$ to $\left.10^{-4} \mathrm{M}\right)$ or ROS scavenger N-acetylcysteine (NAC, $\left.10^{-3} \mathrm{M}\right)$ during H/R. ACh $\left(10^{-5} \mathrm{M}\right)$ was also used in combination with atropine $\left(10^{-4} \mathrm{M}\right.$, a non-selective muscarinic receptor antagonist) or hexamethonium $\left(10^{-4} \mathrm{M}\right.$, a non-selective nicotinic receptor antagonist). The following experimental groups were included: control (Con, normoxia), H/R group (H/R, 12-h hypoxia and 2-h reoxygenation), NAC group (NAC, H/R + $\mathrm{NAC}$ ), ACh group (ACh, H/R + ACh), atropine group (Atro, $\mathrm{H} / \mathrm{R}+\mathrm{ACh}+$ atropine) and hexamethonium group (Hex, H/R +ACh + hexamethonium). These agents were all purchased from Sigma. 


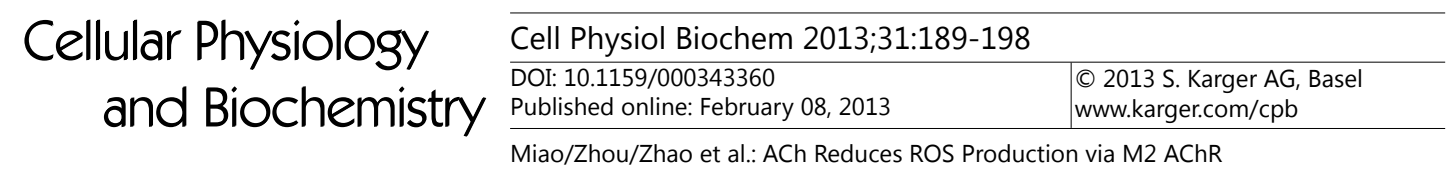

Assay for ROS

H9c2 cells $\left(5 \times 10^{4}\right.$ per well) were seeded in 24-well plates and incubated with $10 \mathrm{mM}$ of 2', $7^{\prime}$-dichlorofluorescin diacetate (DCFH-DA; Beyotime) for $30 \mathrm{~min}$ at $37^{\circ} \mathrm{C}$ in the dark, followed by washing with pre-warmed serum-free DMEM at least three times. The fluorescence of the cells was measured with an inverted fluorescence microscope (TE-2000U, Nikon).

Determination of malondialdehyde (MDA)

The oxidative stress was measured using a Lipid Peroxidation MDA Assay Kit (Beyotime) according to the manufacturer's instructions. The absorbance was read at $544 \mathrm{~nm}$ on the POLARstar OPTIMA multidetection microplate reader (BMG Labtech). The contents of MDA were expressed fold to the control group.

Lactate dehydrogenase release assay

Cell injury was evaluated by the amount of lactate dehydrogenase (LDH) released from cells using the automated chemistry analyzer (Olympus AU2700). The data presented are the results from four independent experiments and the error bars indicate the SEM.

\section{Western blot analysis}

Protein levels were analyzed in whole cell lysates obtained using Cell lysis buffer for Western and IP (Beyotime) and $50 \mu \mathrm{g}$ of samples were resolved on a SDS polyacrylamide gel. Gels were then analyzed by Western blot with the following antibodies: GAPDH Mouse Monoclonal Antibody (Sinopept), mAChR M2 pAb, caspase-3 pAb (Bioworld Technology), rac 1 pAb (Cell Signaling Technology).

\section{Detection of mtROS}

To test the levels of mtROS, MitoSOX Red, a fluorochrome specific to anion superoxide produced in the inner mitochondrial compartment (Invitrogen) was used. Briefly, H9c2 cells were incubated with MitoSox for $0.5 \mathrm{~h}$ at $37^{\circ} \mathrm{C}$, washed twice in PBS, fixed with $4 \%$ paraformaldehyde in PBS for $0.5 \mathrm{~h}$ at room temperature, and washed twice with PBS, incubated for $0.5 \mathrm{~h}$ at $37^{\circ} \mathrm{C}$ with MitoTracker Green (Beyotime) to label mitochondria, and washed again before mounting. MitoSOX intensity was monitored by Nikon TE2000U fluorescence microscopy.

\section{Genomic DNA extraction and real-time PCR}

Since mitochondrial DNA (mtDNA) is a main target of ROS and a reflection of ETC [14], mtDNA copy number was detected using real-time PCR. Genomic DNA was extracted using TIANamp Genomic DNA Kit (Tiangen) according to the manufacturer's instructions. Real-time quantitative PCR was performed by iQ5 (BIO-RAD) with SYBR Premix Ex Taq ${ }^{\mathrm{TM}}$ II (TaKaRa) for D-loop DNA quantitation, and gene expression was normalized by 18sRNA. The following primers (GenScript) were used: D-loop forward: 5'-TGG TTC ATC GTC CAT ACG TT-3', reverse: 5'-TGA CGG CTA TGT TGA GGA AG-3'; 18sRNA forward: 5'-CAT TCG AAC GTC TGC CCT ATC -3', reverse: 5'-CCT GCT GCC TTC CTT GGA-3'.

\section{Detection of XO and NOX activity}

XO and NOX activity were determined by using xanthine oxidase and NADPH oxidase activity assay kits (GENMED) according to the manufacturer's instruction. The change of absorbance at $340 \mathrm{~nm}$ or $510 \mathrm{~nm}$ was monitored by the POLARstar OPTIMA multidetection microplate reader (BMG Labtech), respectively. Protein concentration was measured by Bradford Protein Assay Kit (Beyotime).

SiRNA knockdown of M2 AChR

For knock down of M2 AChR in H9c2 cells, we used small interfering RNA (siRNA) with the rat specific sequence 5'-GUG CUC AUC AAU ACU UUC UTT-3'and the negative control (NC) siRNA sequence 5'-UUC UCC GAA CGU ACG UTT-3' (ABGENT). Transfections were performed using TurboFect siRNA Transfection Reagent (Themo Fisher Scientific) according to the manufacturer's instructions with $100 \mathrm{nM}$ of each siRNA. $48 \mathrm{~h}$ after transfection, the efficiency of siRNA-mediated M2 AChR knockdown was determined by Western blot. The effects of knockdown of M2 AChR on cell injury were tested by MTT (Sigma) cell viability assay. 
Fig. 1. ACh concentrationdependently attenuates H/Rinduced ROS burst. (A, B) Time course of ROS in H9c2 cells during H/R. After 12-h hypoxia and reoxygenation for $1 \mathrm{~h}, 2 \mathrm{~h}, 4 \mathrm{~h}, 8$ h, $16 \mathrm{~h}$ or $24 \mathrm{~h}$ respectively, ROS levels were assayed using DCFHDA. (C, D) ACh reduced the levels of ROS in a concentration-dependent manner. Data are presented as means \pm SEM. $\mathrm{n}=4 .{ }^{*} P<0.01$ vs control group; ${ }^{\#} P<0.01$ vs H/R 2 h group; ${ }^{\&} P<0.01$ vs H/R group. Scale bar $=50 \mu \mathrm{m}$.

A

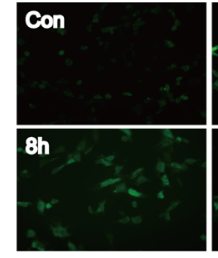

B

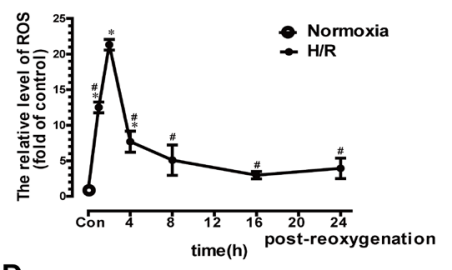

D
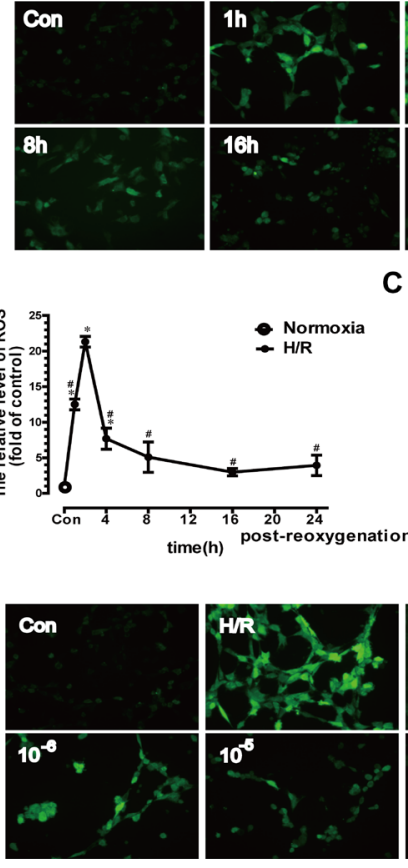

C
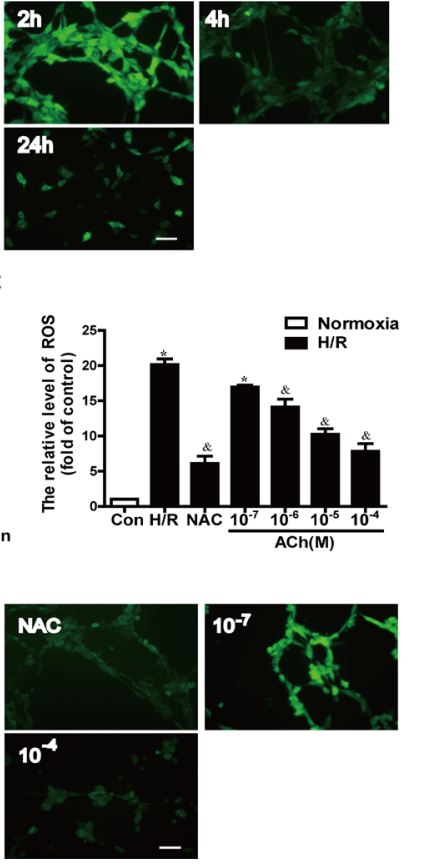

\section{Statistical analysis}

All results are expressed as means \pm SEM. $P<0.05$ was considered statistically significant. Comparisons of results were performed student's t-test or one-way analysis of variance followed by a Tukey post hoc test for multiple comparisons. The package GraphPad Prism Version 5.01 (GraphPad Software Inc) was used to perform all the statistical analysis.

\section{Results}

ACh decreases ROS levels during $H / R$ in a concentration-dependent manner

In order to investigate the optimal time of reoxygation for redox study, H9c2 cells were subjected to $12 \mathrm{~h}$ of hypoxia followed by $1 \mathrm{~h}, 2 \mathrm{~h}, 4 \mathrm{~h}, 8 \mathrm{~h}, 16 \mathrm{~h}$ or $24 \mathrm{~h}$ of reoxygenation respectively. The DCFH-DA was used to monitor intracellular ROS production. As shown in Fig. 1A, B, the levels of ROS elevated rapidly and remarkably reached peak level at $2 \mathrm{~h}$ after reoxygenation. Based on these, further tests were performed using $12 \mathrm{~h}$ of hypoxia followed by $2 \mathrm{~h}$ of reoxygenation.

To determine whether ACh prevents H/R-induced ROS burst, we treated cells with different concentrations of ACh $\left(10^{-7}-10^{-4} \mathrm{M}\right)$ during H/R. NAC $\left(10^{-3} \mathrm{M}\right)$ was used as an antioxidant to neutralize the intracellular ROS. Compared with the H/R group, cells treated with ACh $\left(10^{-6}-10^{-4} \mathrm{M}\right)$ showed a decrease in fluorescence intensity in a concentrationdependent manner (Fig. 1C, D). Further tests were performed using ACh at a concentration of $10^{-5} \mathrm{M}$.

\section{ACh suppresses cell injury and the levels of ROS via M AChR}

To investigate which kind of acetylcholine receptors play the predominant role in AChmediated suppression of cell injury and the level of ROS, atropine $\left(10^{-4} \mathrm{M}\right)$ or hexamethonium $\left(10^{-4} \mathrm{M}\right)$ were applied co-treatment with ACh $\left(10^{-5} \mathrm{M}\right)$ during H/R. LDH release and cleaved caspase-3 were measured to estimate cell injury. ACh inhibited H/R-induced LDH release and decreased cleaved caspase- 3 expression (Fig. 2A, B). The protective effect of ACh was blocked by atropine, while co-treatment with hexamethonium showed no difference compared with ACh group. 
Fig. 2. Involvement of $M A C h R$ in ACh-mediated cardioprotective and antioxidant effect in $\mathrm{H} 9 \mathrm{c} 2$ cells. (A) LDH release assay of H9c2 cells from control (Con), H/R, ACh, ACh-cotreatment with atropine (Atrop) or hexamethonium (Hex) group. (B) Western blot analysis for cleaved caspase-3. (C, D) Role of M AChR in ACh-mediated suppression of ROS. (E) MDA content assay. Data are presented as means \pm SEM. $\mathrm{n}=4 .{ }^{* *} P<0.01$ vs control group; ${ }^{\#} P<0.05$ and ${ }^{\# \#} P<0.01$ vs H/R group; ${ }^{\&} P<0.05$ and ${ }^{\& \&} P<0.01 v s$ ACh group. Scale bar $=50 \mu \mathrm{m}$.

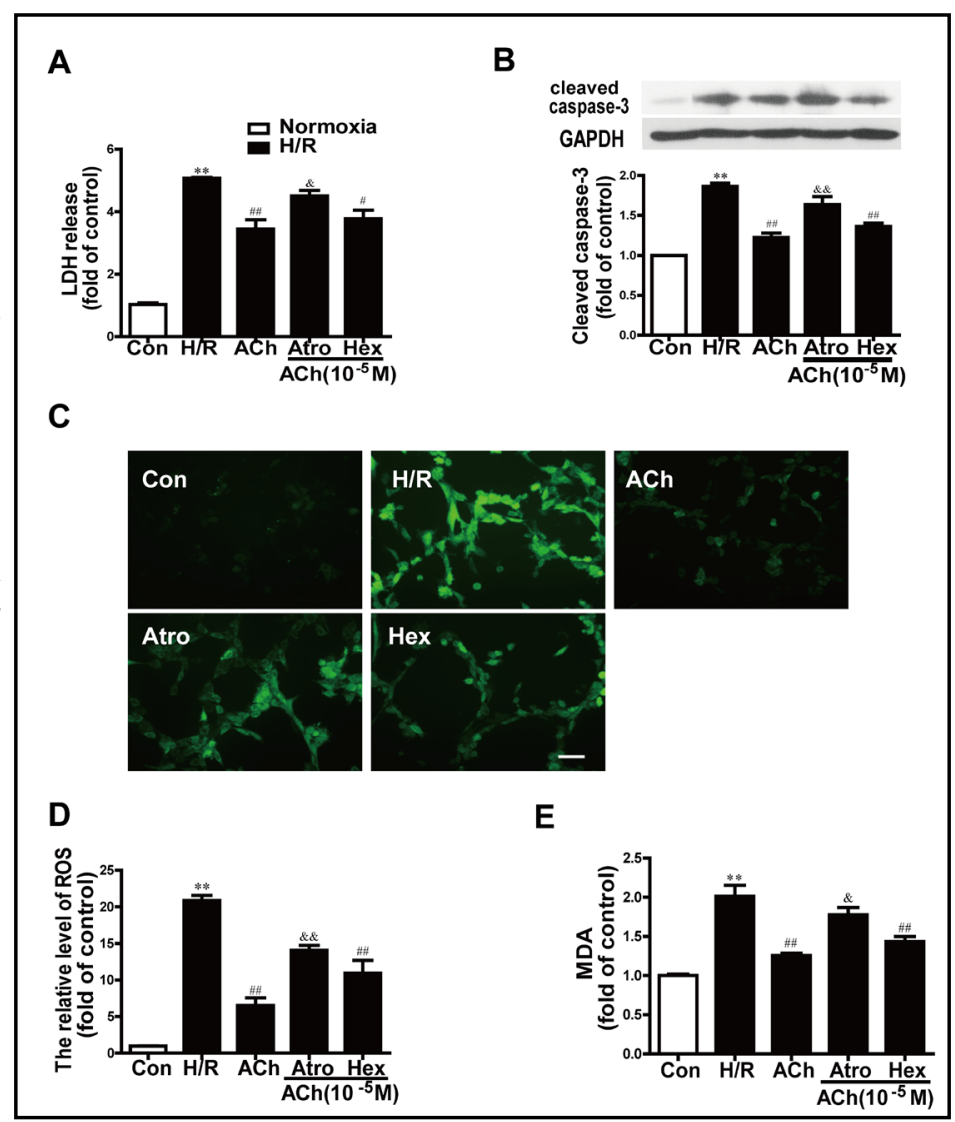

Fig. 3. ACh-mediated mtROS surppression is abolished by atropine. (A) For mtROS assay, the MitoSOX Red fluorescence was colocalized with MitoTracker Green. Shown are representative fluorescence microscopy images. (B) The mean MitoSox Red fluorescence (IOD) of part (A). (C) mtDNA copy number was quantified by comparing D-loop expression to 18sRNA content using real-time PCR. Data are presented as means \pm SEM. $\mathrm{n}=4$. ${ }^{* *} P<0.01$ vs control group; ${ }^{\#} P<$ 0.05 and ${ }^{\# \# ~} P<0.01$ vs H/R group; ${ }^{\&} P<0.05$ and ${ }^{\&} P<0.01$ vs ACh group. Scale bar $=50 \mu \mathrm{m}$.

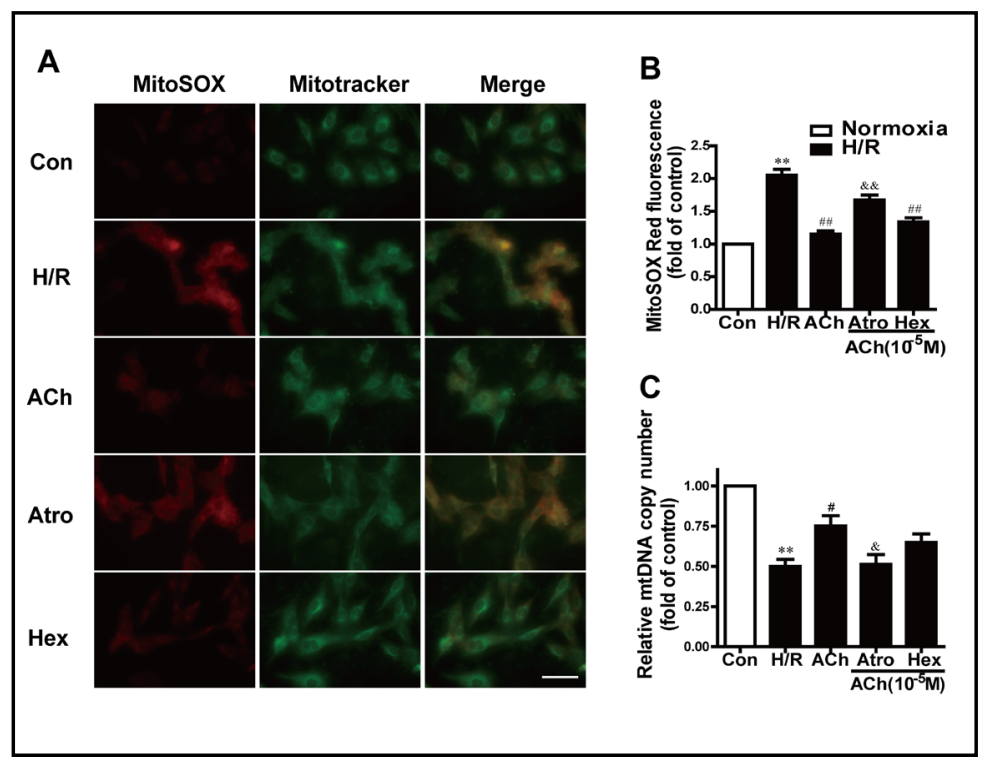

Notably, there is a significant difference in ROS levels between atropine and ACh group, while hexamethonium group did not alter the ROS contents (Fig. 2C, D). In order to further assess the antioxidant effect of ACh, the common marker of oxidative stress MDA was estimated. The content of MDA was also higher in the atropine group compared with the ACh group (Fig. 2E). These data suggests a likely role of the $\mathrm{M}$ receptors in the ACh-induced reduction of cell injury and ROS levels. 

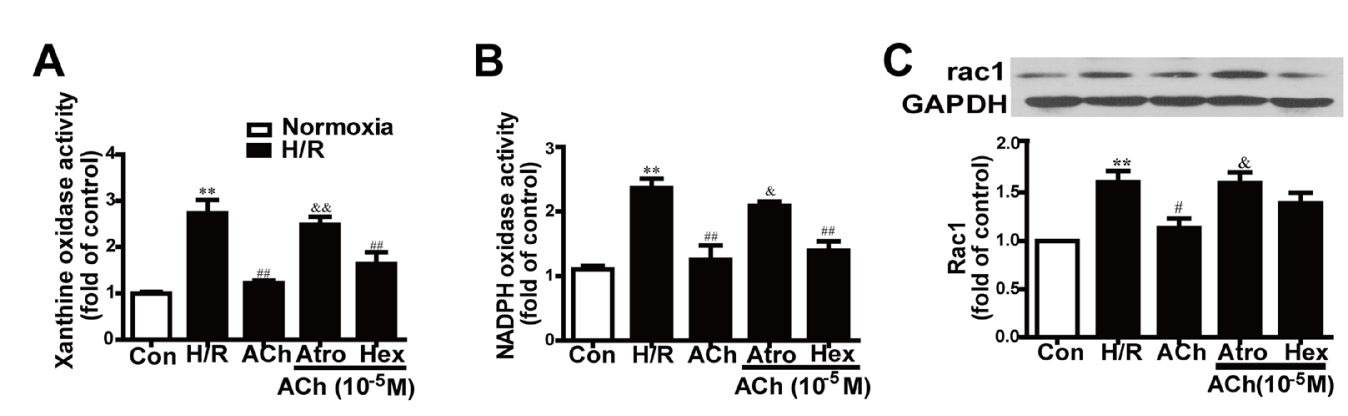

Fig. 4. ACh reduces cytoplasmic ROS via M AChR. (A)XO activity. (B)NOX activity. (C) Expression of rac 1 was analysed by Western blot. Data are presented as means \pm SEM. $\mathrm{n}=4 .{ }^{* *} P<0.01$ vs control group; ${ }^{*} P<0.05$ and ${ }^{\# \#} P<0.01$ vs $\mathrm{H} / \mathrm{R}$ group; ${ }^{\circledR} P<0.05$ and ${ }^{\& \&} P<0.01$ vs ACh group. Scale bar $=50 \mu \mathrm{m}$.

Fig. 5. Role of M2 AChR on the levels of ROS in the presence of ACh during H/R. (A) The silencing efficiency of M2 AChR siRNA were detected by Western blotting, equal protein loading was confirmed with the GAPDH antibody. (B) The effects of knockdown of M2 AChR on cell injury were assayed by MTT. (C, D) M2 AChR small siRNA transfection increases the contents of ROS in the presence of ACh during $H / R$. All the transfection groups were exposed to $\mathrm{H} / \mathrm{R}$ with ACh followed by DCFH-DA staining $48 \mathrm{~h}$ after transfection. Data are presented as means \pm SEM. $\mathrm{n}=4 .{ }^{* *} P<0.01$ vs $\mathrm{NC}$ siRNA group; ${ }^{\#} P<0.01$ vs NC siRNA exposed to HR with ACh group. Scale bar $=50 \mu \mathrm{m}$.

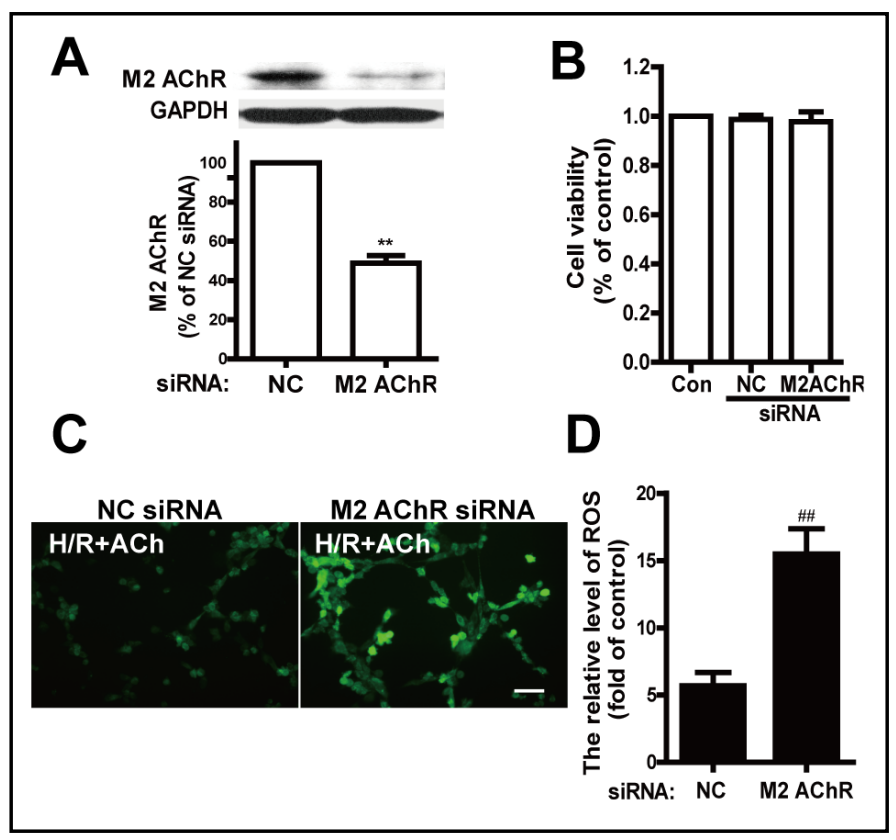

\section{ACh reduces mtROS via $M$ AChR}

Since ACh may decrease the ROS via M receptors, we next presumed that ACh exerts its antioxidant effect by affecting ROS synthesis. We used mitoSOX Red to test mitochondrial superoxide formation. Compared with control, H/R group showed higher mtROS levels. ACh markedly reduced H/R- induced mtROS (Fig. 3A, B), and this effect was prevented by atropine but not hexamethonium. In addition, mtDNA copy number, which is a reflection of ETC function [14], was determined by real time PCR. Compared with H/R group, ACh abolished the decline of mtDNA copy number (Fig. 3C). In addition, atropine rather than hexamethonium displayed lower mtDNA copy number compared with ACh group. These observations indicate that ACh prevents mitochondrial-derived ROS synthesis mainly through $\mathrm{M}$ receptors.

\section{ACh reduces cytoplasmic ROS via M AChR}

Next, the activity of XO and NOX were detected to evaluate cytoplasmic ROS production. ACh prominently eliminated H/R-induced activation of XO and NOX (Fig. 4A, B). Co-treatment with atropine abolished the effect of ACh. Besides, we tested the expression of rac 1, which plays a critical role in NOX activation [15]. Results showed the similar trends (Fig. 4C). These data suggests that ACh inhibits XO and NOX-derived ROS formation mainly through $M$ receptors. 
Fig. 6. The role of $M 2$ AChR in ACh-mediated inhibition of ROS production. Cells were exposed to $\mathrm{H} / \mathrm{R}$ with ACh $48 \mathrm{~h}$ after transfection with M2 AChR or NC siRNA. (A, B) mtROS assay was perfermed using mitosox Red colocalized with MitoTracker Green. (C) mtDNA copy number was quantified by realtime PCR. (D) XO activity. (E) NOX activity. Data are presented as means \pm SEM. $\mathrm{n}=4 .{ }^{* *} P<0.01$ vs NC siRNA group. Scale bar $=50 \mu \mathrm{m}$.

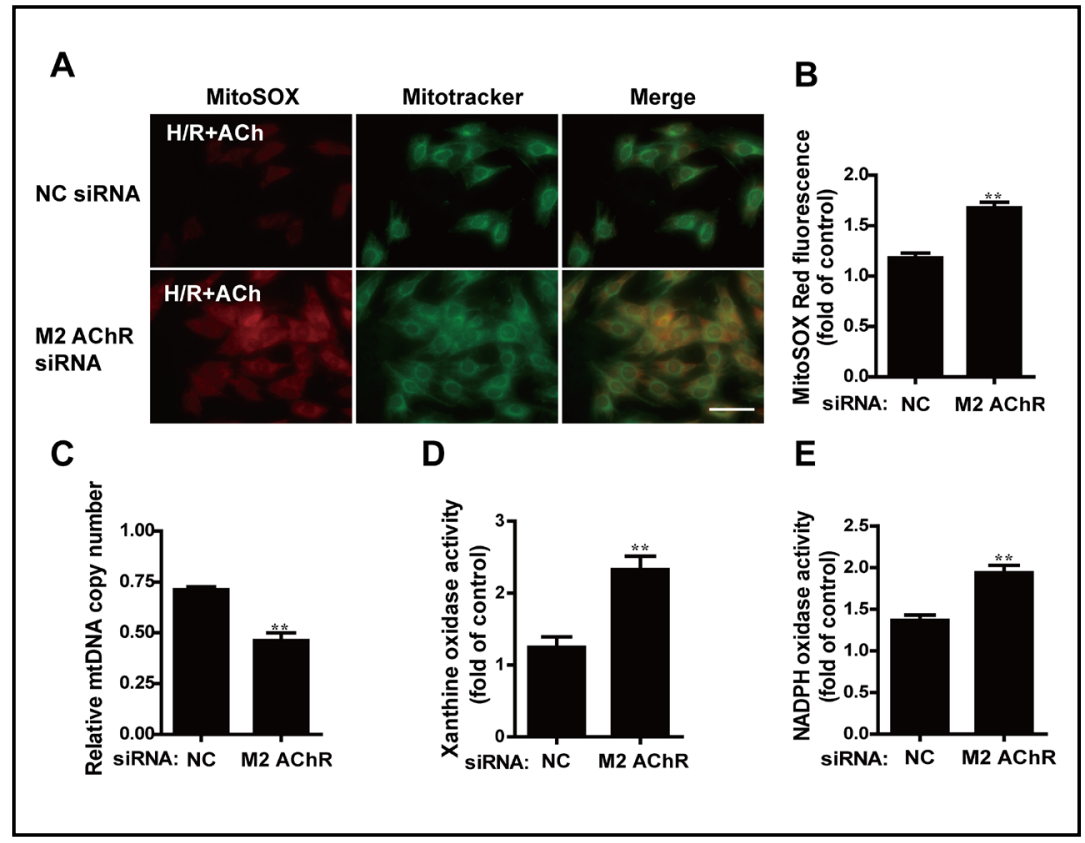

Knockdown of M2 AChR by siRNAs blocks the antioxidant effect of ACh

Upon our previous studies, M2 AChR seems to play a predominant role in ACh-mediated protection in H9c2 cells [13]. RNA interference was performed to knockdown M2 AChR expression. As shown in Fig. 5A, B, compared to the NC siRNA group, M2 AChR siRNA reduced the levels of $\mathrm{M} 2 \mathrm{AChR}$ expression by $51 \%$ and had no significant effect on cell injury. Further, H9c2 cells transfected with M2 AChR siRNA or NC siRNA were exposed to H/R with administration of ACh $48 \mathrm{~h}$ after transfection. Despite the presence of ACh, cells transfected with M2 AChR siRNA displayed higher ROS level compared with the NC siRNA group (Fig. 5C, D), suggesting that M2 AChR participates in the ACh-mediated antioxidant effect.

\section{M2 AChR siRNA increases mitochondrial and cytoplasmic ROS production}

To investigate the role of M2 AChR in different ROS sources, M2 AChR or NC siRNA transfection were performed, and H9c2 cells were exposed to H/R with ACh $48 \mathrm{~h}$ after transfection. Compared with the NC siRNA group, transfection of M2 AChR siRNA markedly increased mtROS and reduced mtDNA copy number (Fig. 6A, B, C). Besides, the XO and NOX activity were also significantly higher in M2 AChR siRNA group than those of NC siRNA group (Fig. 6D, E). All these findings support that M2 AChR played a predominant role in AChmediated inhibition of mitochondrial and cytoplasmic ROS formation.

\section{Discussion}

Increasing evidence has shown that enhancing ACh reduces hypoxia- or ischemiainduced apoptosis and ROS in vivo and in vitro $[10,11,13]$. However, there is little information about whether and how ACh decreases ROS formation during H/R. Here, to our knowledge, we show for the first time that ACh diminishes H/R-induced mitochondria-, XO-, and NOXderived ROS formation via M2 AChR. This is strongly supported by the findings that (1) ACh treatment markedly reduces the contents of ROS in a concentration-dependent manner; (2) ACh dramatically diminishes the level of mtROS, XO and NOX activity; and (3) the above events were blocked by atropine or M2 AChR siRNA transfection. Our findings represent a significant addition to the understanding of ACh-mediated cardioprotection. 
Recently, growing evidence has shown that vagus nerve withdrawl is typically associated with many cardiovascular diseases [16]. In the past decades, VNS has shown promise against heart failure, ventricular tachycardia, and I/R in clinical and experimental studies $[9,17,18]$. However, the precise mechanism(s) remain unclear. The negative inotropic effect reducing myocardial energy consumption can partly explain the cardioprotection of VNS. Meanwhile, several studies, including ours, have attributed cardioprotective effects of VNS or ACh largely to its anti-inflammatory and anti- apoptotic property $[9,19,20]$. Additionally, ACh attenuates hypoxia, $\beta$-adrenoceptor, or angiotensin II-induced ROS [10-13], demonstrating its antioxidant property. Therefore, it is conceivable that ACh possesses a similar effect for attenuating the level of ROS in cardiomyocytes exposed to H/R.

Since there is little information for the time-course change of ROS in cultured cardiomyocytes during reoxygenation, here, we first determined the optimal time of reoxygenation to apply for redox studies in the rat $\mathrm{H} 9 \mathrm{c} 2$ cardiomyocyte cells. In the present study, H9c2 cells were exposed to H/R to mimic I/R. The time of hypoxia was selected according to preliminary trial to induce a significant reduction in cell viability (data not shown). Then, cells were exposed to reoxygenation for $1 \mathrm{~h}, 2 \mathrm{~h}, 4 \mathrm{~h}, 8 \mathrm{~h}, 12 \mathrm{~h}$ or $24 \mathrm{~h}$ respectively. We showed that ROS increased rapidly and reached to the peak at $2 \mathrm{~h}$ after reoxygenation. Based on these results, further studies were performed using 12-h hypoxia followed by 2-h reoxygenation. Next, we examined the antioxidant effect of ACh in H9c2 cells exposed to H/R. ACh decreased the content of ROS in a concentration-dependent manner, accompanied with reduced LDH release and cleaved caspase- 3 expression, suggesting that abrogation of ROS overproduction may be involved in ACh-mediated cardioprotection during H/R.

In view of the detrimental role of excess ROS production in cardiovascular diseases, it is believed that if one can modulate the production of ROS, then the ROS-induced effects of I/R could be alleviated [21]. In general, two kinds of strategies reducing the ROS levels were considered to be available. One is using antioxidants to "clear" ROS. Unfortunately, the functions of antioxidant are very complicated and the results from clinical trial are disappointing [5]. The other is to "inhibit" ROS formation, which is regarded to be more promissing. There are two main categories of ROS sources: mitochondrial and cytosolic. Mitochondria produce ROS mainly through a single electron transport to molecular oxygen in the ETC. In the cytoplasm, NOX and XO are the main sources of ROS formation [22]. Numerous studies have shown that inhibiting these pathways by pharmacological strategies exhibits beneficial effect in animal or in vitro experiments [5]. However, to date many of these inhibitors have not been employed in patients due to their toxcity. Therefore, more effective and feasible modality of therapy is expected. Since we had demonstrated ACh reduces the level of ROS, it is speculated that ACh may attenuate ROS formation through at least one of these resources. Results showed that ACh markedly reduced the mtROS, recovered the mtDNA copy number, decreased XO and NOX activity as well as rac 1 expression, compared with the H/R group. All these data suggest that ACh can reduce mitochondrial-, XO- and NOXderived ROS formation, thus protecting H9c2 cells against H/R.

Since ACh acts on its receptors thereby exerting cardioprotective effects, we next attempt to clarify the receptors involved in H9c2 cells. Five M AChR subtypes (M1-5) [23] and several subunits of nicotinic receptors including $\alpha 7$-nicotinic acetylcholine receptor ( $\alpha 7 \mathrm{nAChR}$ ) [24] have been identified in cardiomyocytes. Pharmacologic evidence indicates that most functional responses are associated with activation of M2 AChR, which is predominant in M AChR in the heart [25]. Recently, a growing body of evidence indicates that $\alpha 7 \mathrm{nAChR}$ is involved in the cholinergic anti-inflammatory pathway and has become a novel therapeutic target for cardiovascular diseases [26]. Previous studies in our laboratory showed the expression of M2 AChR and $\alpha 7 \mathrm{nAChR}$ increase after I/R in the rat isolated heart, indicating the potential role of these receptors in I/R injury [24]. These raise the interesting question that which receptor plays a predominant role in ACh- mediated attenuation of ROS production in cardiomyocytes. Therefore, we used atropine or hexamethonium co-treated with ACh, and found that compared with ACh-treated group, atropine but not hexamethonium augmented mtROS, XO and NOX activity as well as cell injury. Moreover, knockdown of M2 
Fig. 7. Proposed schematic illustration of the mechanism by which ACh exerts its protective effects against $H / R$ injury in this study. During H/R, dysfuntion of ETC and abnormalities of NOX and $\mathrm{XO}$ activity resulted in mitochondrial and cytosolic ROS overproduction, leading to oxidative stress and serious cell injury. ACh reduces oxidative stress by inhibiting these three ROS generation pathways via M2 AChR, thereby protecting H9c2 cells against $\mathrm{H} / \mathrm{R}$ injury.

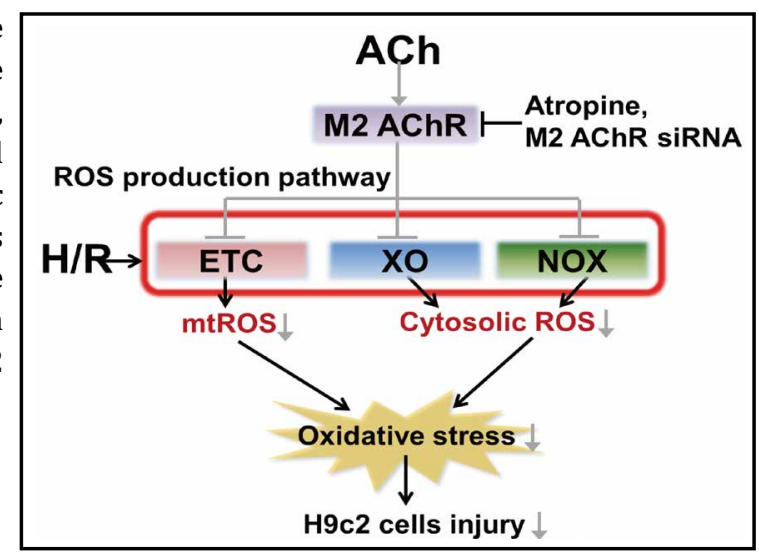

AChR in H9c2 cells howed the similar phenomenon. This is in agreement with our previous study documenting that M2 AChR is involved in the anti-inflammatory property of ACh in H9c2 cells exposed to hypoxia [13]. Taken together, our results suggest that the M2 AChR rather than $\alpha 7 \mathrm{nAChR}$ plays a pivotal role in the ACh-mediated antioxidant as well as antiinflammatory property in H9c2 cells. Further studies of signal transduction pathway linking M2 AChR stimulation to the three ROS formation resources are needed.

In conclusion, ACh inhibits mitochondrial-, $\mathrm{XO}$ - and NOX-derived ROS generation, thus protecting H9c2 cells against H/R-induced cell injury (Fig. 7). M AChR, especially M2AChR play the critical role in antioxidant effects of ACh. Our findings provide new insights into the mechanism underlying ACh-mediated cardioprotection, indicating that increasing ACh release may be a prospective strategy to prevent I/R injury.

\section{Acknowledgements}

This work is supported by Major International (Regional) Joint Research Project of National Natural Science Foundation of China (No. 81120108002), National Natural Science Foundation of China (No. 30930105; No. 30873058; No. 81170176), CMB Distinguished Professorships Award (No. F510000/G16916404) and Natural Science Foundation of Shaanxi Province (No. 2012JZ4001).

\section{References}

$\rightarrow$ Eltzschig HK, Eckle T: Ischemia and reperfusion-from mechanism to translation. Nat Med 2011;17:13911401.

2 Murphy E, Steenbergen C: Mechanisms underlying acute protection from cardiac ischemia -reperfusion injury. Physiol Rev 2008;88:581-609.

3 Minamino T: Cardioprotection from ischemia/reperfusion injury. Circ J 2012;76:1074-1082.

- Radak Z, Zhao Z, Goto S, Koltai E: Age-associated neurodegeneration and oxidative damage to lipids, proteins and dna. Mol Aspects Med 2011;32:305-315.

-5 Chen AF, Chen DD, Daiber A, Faraci FM, Li H, Rembold CM, Laher I: Free radical biology of the cardiovascular system. Clin Sci (Lond) 2012;123:73-91.

-6 Zweier JL, Talukder MA: The role of oxidants and free radicals in reperfusion injury. Cardiovasc Res 2006;70:181-190.

7 Qin F, Simeone M, Patel R: Inhibition of NADPH oxidase reduces myocardial oxidative stress and apoptosis and improves cardiac function in heart failure after myocardial infarction. Free Radic Biol Med 2007;43:271-281. 


\section{Cellular Physiology Cell Physiol Biochem 2013;31:189-198

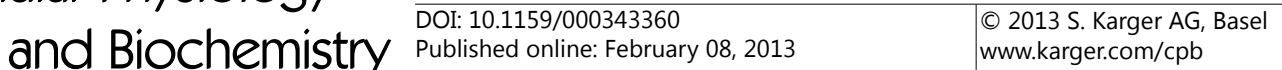 \\ Miao/Zhou/Zhao et al.: ACh Reduces ROS Production via M2 AChR}

8 Uemura K, Li M, Tsutsumi T, Yamazaki T, Kawada T, Kamiya A, Inagaki M, Sunagawa K, Sugimachi M: Efferent vagal nerve stimulation induces tissue inhibitor of metalloproteinase-1 in myocardial ischemiareperfusion injury in rabbit. Am J Physiol Heart Circ Physiol 2007;293:H2254-H2261.

9 Calvillo L, Vanoli E, Andreoli E, Besana A, Omodeo E, Gnecchi M, Zerbi P, Vago G, Busca G, Schwartz PJ: Vagal stimulation, through its nicotinic action, limits infarct size and the inflammatory response to myocardial ischemia and reperfusion. J Cardiovasc Pharmacol 2011;58:500-507.

10 Tsutsumi T, Ide T, Yamato M, Kudou W, Andou M, Hirooka Y, Utsumi H, Tsutsui H, Sunagawa K: Modulation of the myocardial redox state by vagal nerve stimulation after experimental myocardial infarction. Cardiovasc Res 2008;77:713-721.

11 Kim MH, Kim MO, Heo JS, Kim JS, Han HJ: Acetylcholine inhibits long-term hypoxia- induced apoptosis by suppressing the oxidative stress-mediated MAPKs activation as well as regulation of Bcl-2, c-IAPs, and caspase-3 in mouse embryonic stem cells. Apoptosis 2008;13:295-304.

12 Liu JJ, Li DL, Zhou J, Sun L, Zhao M, Kong SS, Wang YH, Yu XJ, Zhou J, Zang WJ: Acetylcholine prevents angiotensin II-induced oxidative stress and apoptosis in H9c2 cells. Apoptosis 2011;16:94-103.

13 Li DL, Liu JJ, Liu BH, Hu H, Sun L, Miao Y, Xu HF, Yu XJ, Ma X, Ren J, Zang WJ: Acetylcholine inhibits hypoxia-induced tumor necrosis factor-alpha production via regulation of MAPKs phosphorylation in cardiomyocytes. J Cell Physiol 2011;226:1052-1059.

14 Tsutsui H, Kinugawa S, Matsushima S: Oxidative stress and mitochondrial DNA damage in heart failure. Circ J 2008;72:A31-A37.

15 Li J, Zhu H, Shen E, Wan L, Arnold JM, Peng T: Deficiency of rac1 blocks NADPH oxidase activation, inhibits endoplasmic reticulum stress, and reduces myocardial remodeling in a mouse model of type 1 diabetes. Diabetes 2010;59:2033-2042.

16 Thayer JF, Yamamoto SS, Brosschot JF: The relationship of autonomic imbalance, heart rate variability and cardiovascular disease risk factors. Int J Cardiol 2010;141:122-131.

17 Brack KE, Coote JH, Ng GA: Vagus nerve stimulation protects against ventricular fibrillation independent of muscarinic receptor activation. Cardiovasc Res 2011;91:437-446.

18 Sabbah HN: Electrical vagus nerve stimulation for the treatment of chronic heart failure. Cleve Clin J Med 2011;78:S24-S29.

19 Kong SS, Liu JJ, Hwang TC, Yu XJ, Lu Y, Zang WJ: Tumour necrosis factor-alpha and its receptors in the beneficial effects of vagal stimulation after myocardial infarction in rats. Clin Exp Pharmacol Physiol 2011;38:300-306.

20 Kakinuma Y, Ando M, Kuwabara M, Katare RG, Okudela K, Kobayashi M, Sato T: Acetylcholine from vagal stimulation protects cardiomyocytes against ischemia and hypoxia involving additive non-hypoxic induction of HIF-1alpha. Febs Lett 2005;579:2111-2118.

21 Chou HC, Chen YW, Lee TR, Wu FS, Chan HT, Lyu PC, Timms JF, Chan HL: Proteomics study of oxidative stress and Src kinase inhibition in H9c2 cardiomyocytes: a cell model of heart ischemia-reperfusion injury and treatment. Free Radic Biol Med 2010;49:96-108.

22 Afanas'Ev I: ROS and RNS signaling in heart disorders: could antioxidant treatment be successful? Oxid Med Cell Longev 2011;2011:293769.

23 Harvey RD: Muscarinic receptor agonists and antagonists: effects on cardiovascular function. Handb Exp Pharmacol 2012;208:299-316.

24 Li DL, Liu BH, Sun L, Zhao M, He X, Yu XJ, Zang WJ: Alterations of muscarinic acetylcholine receptors-2, 4 and alpha7-nicotinic acetylcholine receptor expression after ischaemia/reperfusion in the rat isolated heart. Clin Exp Pharmacol Physiol 2010;37:1114-1119.

25 Harvey RD, Belevych AE: Muscarinic regulation of cardiac ion channels. Br J Pharmacol 2003;139:10741084.

26 Liu C, Su D: Nicotinic acetylcholine receptor alpha7 subunit: a novel therapeutic target for cardiovascular diseases. Front Med 2012;6:35-40. 